\title{
Coeficientes de proporcionalidade nas valvas atrioventriculares: estudo anatômico dos segmentos valvares em indivíduos normais
}

\author{
Coefficients of proportions of the atrioventricular valves: an anatomical study of valvar segments of \\ normal individuals
}

Natália Martins Magacho de ANDRADE ${ }^{1}$, Eduardo TINOIS ${ }^{2}$, Reinaldo Wilson VIEIRA ${ }^{1}$, Domingo Marcolino BRAILE $^{1}$, Orlando PETRUCCI JUNIOR ${ }^{1}$, Pedro Paulo Martins de OLIVEIRA ${ }^{1}$, Lindenberg da Mota SILVEIRA FILHO $^{1}$

RBCCV 44205-756

Resumo

Objetivo: Descrever as relações anatômicas existentes entre as estruturas das valvas cardíacas, levando em consideração os segmentos dos anéis fibrosos e o volume ventricular esquerdo.

Método: Analisaram-se fotografias digitais de 41 corações oriundos de necropsias feitas pelo Núcleo de Perícias Médico Legais. As fotos foram processadas em software em ambiente MATLAB $^{\circledR}$, que forneceu as medidas de perímetro e área valvares e o volume ventricular esquerdo.

Resultados: A média de idade dos indivíduos necropsiados foi de 33 anos, com 17 anos de desvio-padrão. Testaram-se diversas correlações envolvendo as valvas tricúspide e mitral, sendo encontradas correlações fortemente significativas entre a distância intercomissural (DIt) e o perímetro do anel anterior (PA) na valva tricúspide $(r=0,72 \mathrm{com} p<0,05)$ e entre a distância intercomissural (DIm) e o perímetro do anel posterior (AP) na valva mitral $(r=0,63, p<0,05)$. As proporções entre os parâmetros foram: $\mathrm{PA} / \mathrm{DIt}=1,36 \pm 0,24$; $\mathrm{AP} / \mathrm{DIm}=$ $1,38 \pm 0,16$.

Conclusão: As proporções entre o perímetro do anel anterior (tricúspide), o perímetro do anel posterior (mitral) e suas respectivas distâncias intercomissurais têm elevada significância estatística e podem ser aplicadas como parte das técnicas cirúrgicas de reconstruções valvares.

Descritores: Coração, anatomia \& histologia. Valva Mitral. Valva tricúspide.
1 - Disciplina de Cirurgia Cardíaca, Departamento de Cirurgia, Faculdade de Ciências Médicas - Universidade Estadual de Campinas, Brasil. 2 - Centro de Engenharia Biomédica, Faculdade de Ciências Médicas - Universidade Estadual de Campinas, Brasil.

Endereço para correspondência:

Natália Martins Magacho de Andrade

Departamento de Cirurgia Cardíaca - Caixa Postal no 6111.

CEP: 13083-970.

Campinas -SP - Brasil.

Fone/ Fax: (55) (19) 3788-9442

E-mail: rkv@uol.com.br
Artigo recebido em maio de 2005 Artigo aprovado em setembro de 2005 


\section{Abstract}

Objective: To describe the anatomical relationships that exist between the heart valve structures taking into account the segments of the fibrous annuli and the left ventricular volume

Method: Digital photographs of $\mathbf{4 1}$ hearts from autopsies performed by a coroner's office were analyzed. The photographs were processed using MATLAB ${ }^{\circledR}$ software, which supplies measurements of the valvar perimeter and area and the left ventricular volume.

Results: The average age of the corpses studied was 33 years old (standard deviation \pm 17 years). Several ratios involving the tricuspid and mitral valves were tested, with strongly significant correlations found between the intercommissural distance (ItD) and the perimeter of the

\section{INTRODUÇÃO}

A análise das dimensões das valvas cardíacas evoluiu muito nos últimos anos, acompanhando a introdução da biotecnologia nas pesquisas médicas. Assim, reconstruções tridimensionais utilizando-se equipamentos de ecocardiografia, ressonância nuclear magnética ou de tomografia computadorizada tornaram possível o maior conhecimento e planejamento de técnicas cirúrgicas que contemplassem as doenças valvares. Todavia, o retorno aos estudos anatômicos trouxe achados inesperados e de grande valor científico, comprovando a importância das pesquisas post mortem na cirurgia cardiovascular.

Pesquisas sobre doenças das valvas descrevem que mudanças patológicas podem ser congênitas, relacionadas à idade, adquiridas ou secundárias à dinâmica sangüínea. A medida em que estas envelhecem, acabam por sofrer um processo de expansão e abaulamento excessivo, que geralmente não resultam em insuficiência [1,2]. No caso da insuficiência valvar e decorrente de regurgitação sangüínea, esta pode ser resultante de processos patológicos variados, como a febre reumática, doenças degenerativas ou mesmo endocardite infecciosa, doença aguda causada por microorganismos de alta virulência que culmina com lesões cardíacas graves, bem como de um ou mais componentes da valva (folhetos, anel fibroso, cordas tendíneas, músculos papilares) ou por anormalidades no ventrículo ou no átrio [1,3].

O aumento das câmaras ventriculares tem por finalidade a manutenção da função cardíaca estável frente à determinada injúria. Há um aumento do raio (dilatação) da cavidade, que perde a forma elíptica normal, assumindo configuração esférica. Neste novo formato há aumento da tensão parietal no ápice, de forma a igualá-la aos valores da anterior annulus (PA) of the tricuspid valve $(r=0.72$; $p$ value $<0.05$ ) and between the inter-commissural distance (ImD) and the perimeter of the posterior annulus (PP) of the mitral valve $(r=0.63$; $p$-value $<0.05)$. The proportions between these parameters were PA/ItD $=1.36 \pm 0.24$ and $\mathrm{PP} / \mathrm{ImD}=1.38 \pm 0.16$.

Conclusion: The proportions between the perimeter of the anterior annulus (tricuspid) and the perimeter of the posterior annulus (mitral) and their respective distances have high statistical significance and can be applied during surgery of valvar reconstruction.

Descriptors: Heart, anatomy \& histology. Mitral valve. Tricuspid valve.

região medial, embora ocorra também aumento de seus valores. Com a continuidade do processo de hipertrofia, podem ocorrer diversas alterações genéticas, bioquímicas e estruturais, podendo resultar em disfunção ventricular [4]. Quando este processo é decorrente da insuficiência valvar, após a realização de valvoplastia ou troca da valva, consegue-se obter uma mudança morfológica ventricular, com reversão do processo de remodelagem, redução da esfericidade e melhora da função hemodinâmica, diminuindo consideravelmente o processo de disfunção ventricular [5,6].

A medida do tamanho do anel das valvas depende intrinsecamente do método pelo qual o coração é preservado, pois medidas feitas em anéis de valvas fixadas resultaram em menor tamanho do que em corações frescos. Em condições patológicas, como na regurgitação, a circunferência do anel é significantemente maior. Uma vez que o porcentual de redução do anel fibroso é menor, o valor mínimo, obtido durante a sístole, é relativamente maior do que em peças normais [7].

Quanto às alterações que podem ocorrer nas valvas cardíacas, em especial a insuficiência valvar, diversas técnicas cirúrgicas são propostas para sua correção, e, na maioria delas, a reconstrução do anel valvar é o principal objetivo. Sendo assim, torna-se de suma importância o correto conhecimento da anatomia do anel das valvas, bem como do volume das câmaras ventriculares, o qual influi diretamente no funcionamento pré e pós-operatório destas valvas, podendo contribuir sobremaneira no sucesso das valvoplastias. Assim, o presente trabalho tem por objetivo descrever as relações anatômicas existentes entre as diferentes estruturas das valvas cardíacas, levando em consideração os segmentos dos anéis fibrosos e o volume ventricular esquerdo, propondo subseqüentemente 
coeficientes para confecção de próteses adequadas em valvoplastias.

\section{MÉTODO}

Foram analisados 41 corações de pacientes adultos, com idade superior a 14 anos, de ambos os sexos. As medidas foram feitas a partir de fotos de corações frescos, oriundos de necropsias feitas pelo Núcleo de Perícias Médico Legais.

Os corações oriundos das necropsias sofreram processo de desinserção dos átrios, havendo exposição total das valvas cardíacas. Em seguida, o coração foi posicionado para obtenção de melhores imagens. Foram feitas fotos das valvas com uma câmara digital Kodak EasyShare DX3215 Zoom, fixa em um suporte (tripé) para que a angulação e distância da peça anatômica fossem fixas durante todo o procedimento. Logo após, foram feitos cortes laterais nos ventrículos, visibilizando-se o interior destas câmaras, e novas fotos foram tiradas. Uma escala graduada em milímetros foi colocada ao lado da peça no mesmo plano que se desejava realizar as mensurações. As fotos foram processadas em computador, através de software desenvolvido especialmente para o projeto.

O software foi desenvolvido no ambiente MATLAB ${ }^{\circledR}$ [8] e obteve as medidas do (a):

- Perímetro total dos anéis das valvas tricúspide e mitral;

- Área de cada válvula e área total ocupada pelas mesmas;

- Distância intercomissural das valvas cardíacas;

- Área circular do ventrículo esquerdo em sua meiaaltura;

- Medida do eixo maior do ventrículo esquerdo;

- Perímetro e área das lascínias valvares;

- Volume do ventrículo esquerdo.

Para a realização das medidas, o operador definiu demarcadores na imagem que serviram como parâmetros de entrada para o programa. Uma vez definidos os demarcadores, as medidas foram obtidas pelo software e convertidas da unidade pixel para centímetro. O volume foi obtido aproximando-se o ventrículo por um elipsóide de revolução [9-11].

Os resultados obtidos por meio do software são expostos em tabelas e figuras apresentadas nos resultados.

\section{RESULTADOS}

Foram analisadas fotos digitais correspondentes a 41 necropsias, as quais se deram no período de dezembro de 2003 a abril de 2005. A média de idade foi de 33 anos com desvio-padrão de 17 anos, tendo valor mínimo de 15 anos e máximo de 85 anos. Em cada caso foram obtidos três grupos de imagens, compreendendo as valvas cardíacas, a área da meia altura e o interior da câmara ventricular esquerda (evidenciando o septo e a lascínia anterior da valva mitral). Cada caso foi medido três vezes pelo mesmo observador em momentos distintos (Tabela 1).

Tabela 1. Tabela de caracterização da casuística.

\begin{tabular}{lllll}
\hline Variável & Média & $\begin{array}{l}\text { Desvio } \\
\text { padrão }\end{array}$ & $\begin{array}{l}\text { Valor } \\
\text { Mínimo }\end{array}$ & $\begin{array}{l}\text { Valor } \\
\text { Máximo }\end{array}$ \\
\hline Idade & 33 anos & 17 anos & 15 anos & 85 anos \\
Peso & $72 \mathrm{~kg}$ & $11 \mathrm{~kg}$ & $55 \mathrm{~kg}$ & $100 \mathrm{~kg}$ \\
Altura & $171 \mathrm{~cm}$ & $7 \mathrm{~cm}$ & $145 \mathrm{~cm}$ & $185 \mathrm{~cm}$ \\
IMC & 1,8 & 0,2 & 1,5 & 2,2 \\
\hline
\end{tabular}

\section{- Validação do método}

Inicialmente foi feita a comprovação da acurácia do software criado para obtenção dos valores desejados. Para isso, utilizou-se um padrão quadrangular demarcado sobre papel milimetrado, com dimensões de 2,0 x 2,0 cm, a partir do qual foram feitas fotografias com a mesma câmera e na mesma angulação utilizadas na avaliação necroscópica. A média obtida de dez medidas realizadas em momentos diferentes foi de $4,01 \mathrm{~cm}^{2}$ para a área com desvio-padrão de $0,02 \mathrm{~cm}^{2}$; 0 desvio porcentual em relação ao valor verdadeiro, 4,00 cm², foi de $0,003 \%$. Para o perímetro, a média de dez medidas foi de 8,02 cm com desvio-padrão de $0,03 \mathrm{~cm}$; neste caso, o desvio porcentual foi de $0,002 \%$, comparado com o valor verdadeiro que é $8,00 \mathrm{~cm}$.

\section{- Software aplicado às amostras cardíacas}

1. Valva tricúspide

Os valores médios encontrados para as variáveis com os respectivos desvios-padrão estão na Tabela 2.

Tabela 2. Estatísticas das variáveis obtidas da valva mitral.

\begin{tabular}{lllll}
\hline Variável & Média & $\begin{array}{l}\text { Desvio- } \\
\text { Padrão }\end{array}$ & $\begin{array}{l}\text { Valor } \\
\text { Mínimo }\end{array}$ & $\begin{array}{l}\text { Valor } \\
\text { Máximo }\end{array}$ \\
\hline Área anterior tricúspide & 2,92 & 0,97 & 1,22 & 5,02 \\
Perímetro anterior tricúspide & 4,42 & 0,74 & 3,06 & 5,62 \\
Área septal tricúspide & 1,80 & 0,56 & 0,82 & 3,22 \\
Perímetro septal tricúspide & 3,00 & 0,41 & 2,32 & 4,06 \\
Área posterior tricúspide & 1,51 & 0,45 & 0,63 & 2,67 \\
Perímetro posterior tricúspide & 2,28 & 0,38 & 1,59 & 3,23 \\
Área total tricúspide & 6,28 & 1,79 & 3,17 & 10,08 \\
Perímetro total tricúspide & 9,61 & 1,20 & 7,20 & 12,06 \\
Dist. intercomissural tricúspide & 3,24 & 0,54 & 2,12 & 4,52 \\
\hline
\end{tabular}


Todas as variáveis foram corrigidas por idade, peso e altura. Para correção foi feita regressão múltipla para cada variável em função das co-variáveis (idade, peso e altura), em seguida, realizou-se a normalização para o valor mais baixo das medidas.

Em seguida, testou-se a correlação entre a distância intercomissural e o perímetro do anel anterior da valva tricúspide, obtendo-se coeficiente de correlação $r=0,72$ com $\mathrm{p}<0,05$ (Figura 1 ).

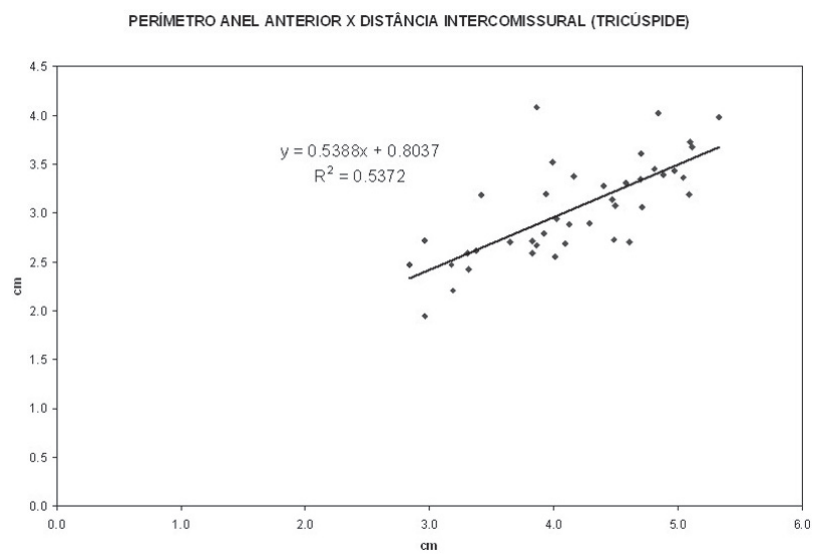

Fig. 1 - Perímetro do anel posterior versus distância intercomissural na valva tricúspide com equação obtida por ajuste de mínimos quadrados.

Dada a correlação significativa entre as variáveis acima, estimou-se a proporção perímetro do anel anterior / distância intercomissural, demonstrados através dos parâmetros estatísticos: média 1,36, desvio-padrão 0,24, valor mínimo 0,92 e valor máximo 1,82.

\section{Valva mitral}

Os valores médios encontrados para as variáveis com os respectivos desvios-padrão estão na Tabela 3.

Tabela 3. Estatísticas das variáveis da valva mitral.

\begin{tabular}{lllll}
\hline Variável & Média & $\begin{array}{l}\text { Desvio- } \\
\text { padrão }\end{array}$ & $\begin{array}{l}\text { Valor } \\
\text { mínimo }\end{array}$ & $\begin{array}{l}\text { Valor } \\
\text { máximo }\end{array}$ \\
\hline Área anterior mitral & 2,94 & 0,73 & 1,71 & 4,88 \\
Perímetro anterior mitral & 4,60 & 0,62 & 3,37 & 5,96 \\
Área posterior mitral & 1,74 & 0,54 & 0,84 & 3,01 \\
Perímetro posterior mitral & 3,47 & 0,46 & 2,75 & 4,55 \\
Área total mitral & 4,72 & 1,21 & 2,59 & 7,76 \\
Perímetro total mitral & 7,92 & 1,16 & 4,20 & 10,13 \\
Dist. intercomissural mitral & 2,46 & 0,35 & 1,80 & 3,16 \\
Área lascínia mitral & 5,92 & 1,26 & 3,95 & 9,63 \\
Perímetro lascínia mitral & 6,75 & 0,89 & 5,14 & 10,63 \\
\hline
\end{tabular}

Em seguida, testaram-se as seguintes correlações entre variáveis:

- Perímetro interno da lascínia X perímetro do anel posterior

- Perímetro total do anel valvar X volume ventricular esquerdo

- Distância intercomissural X perímetro do anel posterior

- Área total da valva X volume ventricular esquerdo

- Eixo maior ventrículo esquerdo X área total da valva

- Área em meia altura do ventrículo esquerdo X área total da valva

Os resultados para os coeficientes de correlação de Pearson para os testes acima apontaram valores elevados de correlação entre distância intercomissural e perímetro no anel posterior na valva mitral $(r=0,63 \mathrm{com} p<0,05)$ - Figura 2 .

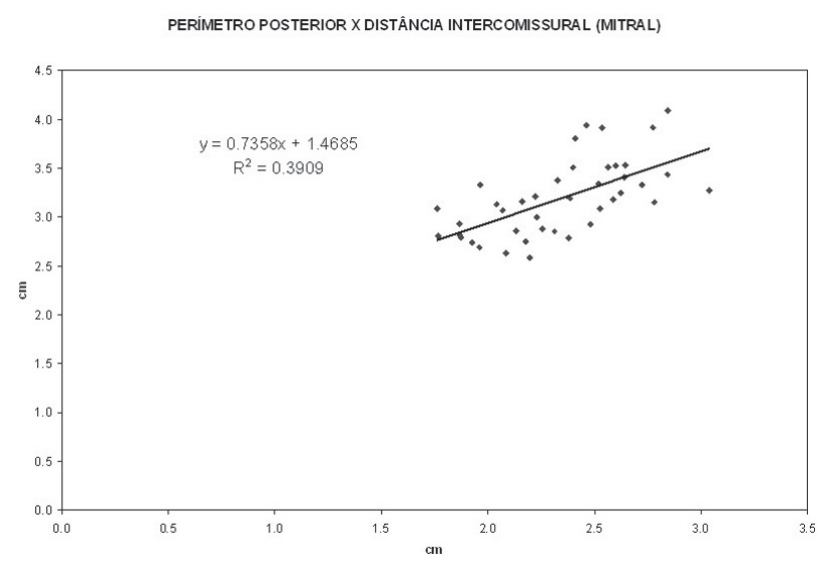

Fig. 2 - Perímetro do anel posterior versus distância intercomissural na valva mitral com equação obtida por ajuste de mínimos quadrados.

Dada a correlação significativa entre as variáveis acima, estimou-se a proporção perímetro do anel posterior/distância intercomissural, demonstrados através dos parâmetros estatísticos: média 1,38, desviopadrão 0,16, valor mínimo 1,08 e valor máximo 1,75.

\section{Ventrículo esquerdo}

Os valores médios encontrados para as variáveis com os respectivos desvios-padrão estão na Tabela 4.

Os parâmetros obtidos nas fotografias do ventrículo esquerdo foram utilizados nas relações com a valva mitral, já demonstradas acima. 
Tabela 4. Estatísticas das variáveis associadas ao ventrículo esquerdo.

\begin{tabular}{llll}
\hline & Média & $\begin{array}{l}\text { Desvio- } \\
\text { padrão }\end{array}$ & $\begin{array}{l}\text { Valor } \\
\text { máximo }\end{array}$ \\
\hline Eixo maior vent. esquerdo & 7,54 & 0,79 & 9,19 \\
Área $1 / 2$ altura vent. esquerdo & 15,41 & 4,41 & 26,77 \\
Perímetro 1/2 altura vent. esquerdo & 14,17 & 1,97 & 19,03 \\
Volume ventrículo esquerdo & 77,67 & 27,16 & 155,08 \\
\hline
\end{tabular}

\section{COMENTÁRIOS}

O padrão ouro utilizado para medida das variáveis é a ecocardiografia. Singh \& Mohan [12] e Ormiston et al. [13] realizaram medidas para algumas das variáveis acima descritas utilizando ecocardiografia. A leitura destes trabalhos mostra que há pequenas diferenças entre as medidas, provavelmente, decorrentes de particularidades dos métodos utilizados. A comparação de nossos resultados mostra concordância com a literatura.

Evangelista et al. [14] publicaram um estudo no qual compararam os valores de áreas valvares de indivíduos não cardiopatas obtidos de estudos necroscópicos e ecocardiográficos, concluindo que a maioria dos valores são similares, sendo, contudo, que a área da valva tricúspide pelo exame ecocardiográfico, em vista apical, tenha se mostrado menor do que a calculada na série necroscópica.

A validação do método, feita pela análise de um objeto padrão com dimensões conhecidas, mostrou que o método é robusto, apresentando pouca variação inerente.

Algumas variáveis, tais como volume do ventrículo esquerdo e área do anel anterior da tricúspide, apresentaram o desvio-padrão maior em relação à média, devendo-se principalmente à grande variabilidade inerente ao ser humano.

Os valores médios dos parâmetros das valvas cardíacas são maiores no sexo masculino em relação ao feminino. Todavia, esta característica torna-se oposta quando se relaciona estes valores com a área de superfície corpórea [15].

Foi feita uma análise para a caracterização das variáveis que influenciam significativamente os valores medidos. Para esta amostra necroscópica, o peso corpóreo dos indivíduos foi o que mostrou maior influência nas medidas obtidas. A área de superfície corpórea e altura mostraram, consecutivamente, muito pouca influência. Mesmo assim, para a matriz inicial de valores paramétricos, foi realizada uma regressão múltipla, eliminando a influência destas variáveis, bem como da idade.

A correlação encontrada na valva tricúspide entre distância intercomissural e perímetro do anel anterior é um resultado que fundamenta muitas técnicas cirúrgicas atualmente empregadas na reconstrução desta valva. A distância intercomissural pode ser facilmente obtida durante o procedimento cirúrgico. Utilizando-se a proporção calculada para indivíduos normais, decrescida de dois desvios-padrão, pode-se inferir um tamanho protético adequado para a reconstrução do anel anterior dilatado na insuficiência tricúspide, com confiança superior a 95\%. O erro-padrão encontrado na proporção entre os parâmetros é significantemente baixo, o que corrobora com a acurácia dos valores obtidos.

Diversos estudos encontrados na literatura preconizam que a área da valva mitral normal varia de 4 (quatro) a 6 (seis) $\mathrm{cm}^{2}$, o que valida os dados encontrados por este estudo $[5,16]$.

Diferentes correlações foram analisadas na tentativa de identificar quais variáveis se relacionam entre si na valva mitral e que podem ser utilizadas como modelo de normalidade na avaliação de estruturas patológicas. A principal correlação encontrada foi entre o perímetro do anel posterior e a distância intercomissural $(\mathrm{r}=0,63)$, embora todas as demais correlações tenham significância estatística por apresentarem $\mathrm{p}<0,05$, com confiança superior a $95 \%$. Não foi observada correlação intensa entre o perímetro da lascínia e o anel posterior $(r=0,26$, com $\mathrm{p}<0,05)$, provavelmente devido ao tipo de incisão anatômica adotada para realização das medidas. No campo cirúrgico, no entanto, a medida do perímetro é feita com o ventrículo preservado, devendo ser testada a correlação em estudo futuro nestas condições.

É consenso que as disfunções valvares relacionadas à cardiomiopatia dilatada estão relacionadas à dilatação de estruturas como o anel fibroso, átrio e ventrículo esquerdos, distensão das cordas tendíneas e disfunção da contração dos músculos papilares e da parede ventricular esquerda. Enfocando as estruturas da valva mitral, muitos autores afirmam que, nas valvas regurgitantes, a distância entre os trígonos fibrosos permanece constante, uma vez que se trata de uma parte do esqueleto fibroso cardíaco, sendo o anel posterior responsável pela dilatação e insuficiência valvar. Em decorrência destes achados, muitas técnicas de valvoplastia foram propostas, como a técnica de Braile et al. [17], utilizada em nosso serviço.

Hueb et al. [18], entretanto, encontraram em análise de corações fixados que há dilatação, tanto das regiões fibrosas, quanto das regiões musculares do anel mitral, sendo assim proporcional e não exclusivamente na porção posterior.

Deste modo, calculou-se a proporcionalidade entre o perímetro do anel posterior e a distância intercomissural, obtendo-se uma significância importante comprovada pelo baixo erro-padrão. Como na valva tricúspide, esta proporção pode ser utilizada na prática cirúrgica para a escolha do 
tamanho da prótese para a reconstrução valvar.

Quanto às medidas obtidas do ventrículo esquerdo, obteve-se um valor compatível com os valores estabelecidos na literatura para volume ventricular. Acredita-se que a cavidade ventricular esquerda do cadáver tenha uma conformação que se aproxima muito mais daquela existente, em vida, no final da sístole do que daquela da diástole. O coração muito raramente pára em sístole, mas sim em um estado hiper-relaxado (mais do que uma diástole fisiológica), ocorrendo, após tempo variável, um processo lento de contratura isquêmica de todo o miocárdio que provoca um grau de redução da cavidade ventricular esquerda muito maior que o de uma sístole fisiológica [12].

Encontraram-se relações menos expressivas, mas ainda significativas, entre o perímetro e a área da valva mitral com o volume ventricular, impossibilitando a utilização das variáveis para inferir o volume.

\section{CONCLUSÃO}

As proporções entre o perímetro do anel anterior (tricúspide), o perímetro do anel posterior (mitral) e suas respectivas distâncias intercomissurais têm elevada significância estatística e podem ser aplicadas como parte das técnicas cirúrgicas de reconstruções valvares.

\section{REFERÊNCIAS BIBLIOGRÁFICAS}

1. Tei C, Pilgrim JP, Shah PM, Ormiston JA, Wong M. The tricuspid valve annulus: study of size and motion in normal subjects and in patients with tricuspid regurgitation. Circulation. 1982;66(3):665-71.

2. Bonow RO, Carabello B, de Leon AC, Edmunds LH Jr, Fedderly BJ, Freed MD et al. ACC/AHA Guidelines for the management of patients with valvular heart disease. A report of American College of Cardiology / American Heart Association. Task Force on Practice (Committee on Management of Patients with Valvular Heart Disease). J Am Coll Cardiol. 1988;7(6):672-707.

3. Keeffe BG, Otto CM. Mitral regurgitation. Minerva Cardioangiol. 2003;51(1):29-39.

4. Zornoff LAM, Cicogna AC, Paiva SAR, Spadaro J: Remodelamento e seu impacto na progressão da disfunção ventricular. Rev Soc Cardiol Estado São Paulo 2002;12(3):371-8.
5. Herregods MC, Tau A, Vandeplas A, Bijnens B, Van De Werf F. Values for mitral valve annulus dimensions in normals and patients with mitral regurgitation. Echocardiography. 1997;14(6 Pt 1):529-34.

6. Radovanovic N, Petrovic LJ, Zorc M, Mihajilovic B, Kovac $\mathrm{M}$, Nicin S et al. Changes in left ventricular morphology and function in end-stage dilated cardiomyopathy after reductive annuloplasty of double mitral and tricuspid orifices. J Card Surg. 2002;17(3):201-4.

7. HauckAJ, Freeman DP, Ackermann DM, Danielson GK, Edwards WD. Surgical pathology of the tricuspid valve: a study of 363 cases spanning 25 years. Mayo Clin Proc. 1988;63(9):851-63.

8. Hanselman D, Littlefield B. MATLAB 6: Curso Completo. Prentice Hall;2003.

9. Jain AK. Fundamentals of digital image processing. Prentice Hall;1989.

10. Hämmerlin G, Hoffmann KH. Numerical mathematics. Springer-Verlag;1991.

11. Bezerra SJ. Um modelo matemático para o cálculo do volume do ventrículo esquerdo. Campinas;1985.

12. Singh B, Mohan JC. Atrioventricular valve orifice areas in normal subjects: determination by cross-sectional and doppler echocardiography. Int J Cardiol. 1994;44(1): 85-91

13. Ormiston JA, Shah PM, Tei C, Wong M. Size and motion of the mitral valve annulus in man. I. a two-dimensional echocardiographic method and findings in normal subjects. Circulation. 1981;64(1):113-20.

14. Evangelista A, del Castillo HG, Gonzalez-Alujas T, GarciaDorado D, Guix M, Soler-Soler J. Normal values of valvular annular areas: comparison of the results of a necropsy and an echocardiographic series. Rev Esp Cardiol. 1996;49(2): 111-6.

15. Kitzman DW, Scholz DG, Hagen PT, Ilstrup DM, Edwards WD. Age-related changes in normal human hearts during the first 10 decades of life. Part II (maturity): a quantitative anatomic study of 765 specimens from subjects 20 to 99 years old. Mayo Clin Proc. 1988;63(2):137-46.

16. Guyton AC, Hall JE. Tratado de fisiologia médica. $10^{\mathrm{a}}$ ed. Rio de Janeiro:Guanabara Koogan;2002. p.232-8.

17. Braile DM, Ardito RV, Pinto GH, Santos JLV, Zaiantchick M, Souza DRS et al. Plástica mitral. Rev Bras Cir Cardiovasc. 1990;5(2):86-98.

18. Hueb AC, Jatene FB, Moreira LF, Pomerantzeff PM, Kallas E, de Oliveira SA. Ventricular remodeling and mitral valve modifications in dilated cardiomyopathy:new insights from anatomic study. J Thorac Cardiovasc Surg. 2002;124(6):1216-24. 